Relations industrielles

Industrial Relations

\title{
Gazaille, Alain, Réaliser l'équité salariale et Chicha, Marie-Thérèse, L'équité salariale : mise en oeuvre et enjeux
}

\section{Jacques Mercier}

Volume 52, numéro 4, 1997

URI : https://id.erudit.org/iderudit/051211ar

DOI : https://doi.org/10.7202/051211ar

Aller au sommaire du numéro

Éditeur(s)

Département des relations industrielles de l'Université Laval

ISSN

0034-379X (imprimé)

1703-8138 (numérique)

Découvrir la revue

Citer ce compte rendu

Mercier, J. (1997). Compte rendu de [Gazaille, Alain, Réaliser l'équité salariale et Chicha, Marie-Thérèse, L'équité salariale : mise en oeuvre et enjeux]. Relations industrielles / Industrial Relations, 52(4), 895-898.

https://doi.org/10.7202/051211ar

Tous droits réservés @ C Département des relations industrielles de l'Universite Laval, 1997
Ce document est protégé par la loi sur le droit d'auteur. L'utilisation des services d'Érudit (y compris la reproduction) est assujettie à sa politique d'utilisation que vous pouvez consulter en ligne.

https://apropos.erudit.org/fr/usagers/politique-dutilisation/ 


\section{Réaliser l'équité salariale}

par Alain GAZAILLE, Montréal : Wilson \& Lafleur, 1996, 154 p., ISBN 2-89127380-X

\section{L'équité salariale: mise en ouvre et enjeux}

par Marie-Thérèse CHICHA, Cowansville : Les éditions Yvon Blais inc., 1997, 240 p., ISBN 2-89451-114-0

La Loi sur l'équité salariale (projet de loi $n^{\circ} 35$ ) présentée le 15 mai 1996 fut adoptée par l'Assemblée nationale le 21 novembre 1996 et sanctionnée le même jour. Ainsi, huit ans après l'Ontario, le Québec se dotait d'une législation dite "proactive " en matière d'équité salariale couvrant tant le secteur public que le secteur privé, dans le cas de tout employeur dont l'entreprise compte 10 salariés ou plus, avec obligation d'établir un ou des programmes d'équité salariale pour les employeurs dont l'entreprise compte 50 salariés ou plus. Obligation est également faite aux employeurs de 100 salariés ou plus de permettre la participation des salariés à l'établissement du ou des programmes en instituant un ou des comités d'équité salariale au sein duquel ou desquels ils sont représentés. Pour les entreprises de moins de 50 salariés, il n'y a qu'une obligation de résultat. Les ajustements salariaux requis pour atteindre l'équité salariale doivent avoir été déterminés ou un programme d'équité salariale doit avoir été complété dans un délai de quatre ans de l'entrée en vigueur du chapitre II de la loi (Modalités d'application). Enfin, une Commission de l'équité salariale est instituée avec pour fonction, entre autres choses, de diffuser l'information destinée à faire comprendre et accepter l'objet et les dispositions de la loi, d'aider à la formation des membres des comités d'équité salariale et de prêter assistance aux entreprises en développant des outils appropriés.

Pour les entreprises concernées qui voudraient déjà aller de l'avant avec l'équité salariale, le premier obstacle est le peu de matériel didactique disponible sauf le texte même de la loi. Ce sera sans doute ce à quoi s'attaquera en priorité la Commission. Entre temps, les deux ouvrages cités en titre peuvent aider les personnes concernées ou intéressées à s'y retrouver. Ils sont de facture et de contenu différents mais leur récente publication justifie de les présenter.

"Réaliser l'équité salariale» d'Alain Gazaille se veut un guide pratique décrivant étape par étape la démarche à suivre pour réaliser l'équité salariale. Le texte est facilement accessible, concis et clair. On y trouve de nombreux schémas servant à présenter la matière ou à récapituler celle-ci. Nombreux sont les ouvrages de ce genre et l'auteur ne cache pas qu'il n'a fait que reprendre des écrits de diverses sources (il n'y a malheureusement pas de bibliographie et les notes infrapaginales citant des sources se comptent sur les doigts de la main) pour en présenter une synthèse. Il y a toutefois guide pratique et guide pratique. L'ouvrage comporte 17 chapitres de sept à huit pages chacun ce qui laissera plus d'une fois le lecteur sur sa faim sur des sujets aussi importants et documentés que les comités d'évaluation des emplois, l'évaluation comme telle, le choix d'un outil d'évaluation, etc., en contexte, faut-il le répéter, d'équité salariale. C'est le "syndrome de l'acétate (ou du transparent)" cher aux consultants avec pour chaque chapitre une page titre, une page table des matières, reprise des titres et soustitres, schémas récapitulatifs ou de présentation et quelques pages, parfois quelques lignes de texte. Comme sa parution a précédé l'adoption de la Loi sur l'équité salariale, c'est au texte du projet de loi que l'auteur a dû se référer en décrivant les différentes étapes de la démarche proposée. Ceci a pour effet de rendre certains passages incomplets ou désuets en regard du texte de la loi étant donné les modifications qu'a subies 
le projet de loi avant d'être adopté et sanctionné. Par exemple, le chapitre 5 sur la détermination des groupes d'emplois devrait être revu et corrigé. On y trouve assimilés emploi et catégorie d'emplois, ce que ne fait pas le texte de la loi (art. 54) ; la liste de facteurs pouvant servir à regrouper les emplois n'a plus rien à voir avec le texte de la loi non plus et l'affirmation que le critère de pourcentage $(60 \%$ pour identifier les catégories d'emplois à prédominance féminine ou masculine) n'est pas suffisant est erronée (art. 55).

Le chapitre 14 comporte aussi des lacunes en rapport avec le texte de la loi. Il traite des méthodes de comparaison des résultats de l'évaluation pour déterminer les écarts salariaux à corriger. L'une des méthodes décrites et qu'on trouvait dans le projet de loi, la méthode dite " courbe à courbe ", ne se retrouve plus dans le texte de la loi. Ce chapitre comporte aussi un exemple erroné d'une exclusion d'un emploi aux fins d'estimer la courbe salariale des catégories à prédominance masculine si l'on a recours à l'approche globale. Le motif d'exclusion est que le salaire pour cet emploi n'est pas représentatif (trop élevé), ce que ne permet pas l'article 62 de la loi.

Réaliser l'équité salariale passe par l'évaluation des emplois et à cet égard l'auteur, pour demeurer pratique, oriente rapidement l'opération par un passage obligé à la méthode dite "des points et facteurs ". Ceci ne fait que refléter la tendance générale d'évaluation des emplois en contexte d'équité salariale ou en général. Ce qui surprend un peu, par contre, c'est le recours à un plan d'évaluation (reproduit en appendice avec la grille de pondération des facteurs) à 16 facteurs, plusieurs étant multidimensionnels, à titre d'exemple. Il est vrai qu'un tel plan a servi d'outil de base dans plusieurs exercices de relativité salariale (et non d'équité salariale comme le laisse entendre l'auteur) dans les secteurs public et parapublic québécois, mais l'une des premières tâches de la Commission sur l'équité salariale sera de vérifier la conformité de tels exercices à la loi incluant la méthode et les outils (art. 119). Comme un exemple devient souvent une référence, une certaine prudence est de mise ici. De pius, le Syndicat canadien de la fonction publique (SCFP), qui a été et demeure le syndicat le plus actif dans le domaine de l'évaluation des emplois en contexte d'équité salariale et qui a été associé de très près à la conception du fameux "plan à 16 facteurs " utilise depuis plusieurs années déjà une version plus simple d'application et de compréhension soit un plan à 12 facteurs unidimensionnels.

Comme l'annonce son titre L'équité salariale: mise en ouvre et enjeux, le livre de Marie-Thérèse Chicha poursuit deux objectifs : présenter les différentes étapes de mise en œuvre de l'équité salariale selon les conditions et les modalités prévues dans la nouvelle Loi sur l'équité salariale et bien faire saisir au lecteur la problématique de l'équité salariale et ses enjeux. C'est pourquoi elle consacre ses deux premiers chapitres aux fondements de l'équité salariale et aux approches pour combattre les inégalités salariales basées sur le sexe. Le lecteur est amené à comprendre pourquoi une loi proactive en la matière s'imposait et l'on sait l'importance de la sensibilisation au phénomène de la discrimination, ici à l'endroit des femmes du fait de la sous-rémunération des emplois à prédominance féminine, pour réaliser l'équité salariale. Pour reprendre les termes de l'auteure "L'idée fondamentale est que l'équité salariale n'est pas seulement, ni principalement une question technique. C'est avant tout une question de justice sociale " (Avant-propos). D'où ces deux premiers chapitres et les nombreux commentaires remettant toujours à l'avantscène la préoccupation sociale à travers tout le reste de l'ouvrage consacré au premier objectif mentionné ci-dessus. C'est un ouvrage bien fait et solide sur les plans de l'analyse et de la démarche. On y retrouve également le texte de la 
loi en annexe, une bibliographie imposante et à jour et un index analytique. Sans ambitionner constituer un guide pratique permettant de répondre sans faille aux diverses questions et aux multiples problèmes d'application rencontrés dans la mise en œuvre de l'équité salariale, l'ouvrage fourmille d'ilustrations, de rêflexions et de commentaires sur tel ou tel aspect de la démarche prévue dans la loi.

Le chapitre 1 offre une bonne synthèse de la littérature sur la mesure de l'écart salarial entre les sexes, les facteurs explicatifs et les points de vue théorique sur le sujet. Il permet entre autres de relativiser les possibilités théoriques de réduction de l'écart par le biais d'une loi proactive sur l'équité salariale. En effet, une faible partie de l'écart, environ le sixième, serait attribuable à ce type de discrimination envers les emplois à prédominance féminine sans compter les limites d'application de la loi (niveau de couverture, approche par entreprise, délais de réalisation, etc.).

Le chapitre 2 dresse un portrait du traitement accordé au problème des inégalités salariales entre emplois à prédominance féminine et emplois à prédominance masculine au Canada et au Québec. Un apport important de ce chapitre réside dans la comparaison des deux grandes approches légales (ou modèles juridiques) en matière de recherche d'équité salariale soit l'approche passive (reposant sur le principe de la plainte) ou traditionnelle et l'approche proactive qui, comme c'est le cas de la nouvelle Loi sur l'équité salariale, pose comme préalable l'existence d'une discrimination systémique dans la rémunération des emplois dits féminins et oblige les employeurs à reconsidérer celle-ci dans une démarche exempte de biais sexistes et à en corriger les écarts. Ce chapitre est aussi l'occasion de présenter l'état de la situation des diverses législations provinciales et fédérales. Il aurait été intéressant d'introduire un troisième modèle ici : celui de la négociation (secteur syndi- qué). On le retrouve en partie sous le titre de " programmes volontaires " à l'intérieur du modèle traditionnel, mais le traitement qu'en fait l'auteure reste prisonnier de la logique de comparaison de deux modèles juridiques. Or la négociation collective, sous la poussée des syndicats de plus en plus sensibilisés aux questions de la condition féminine, a permis à nombre de femmes de réaliser des gains en matière d'équité salariale, principalement mais pas uniquement, dans le secteur public. Ce sont aussi les syndicats et des groupes de femmes qui ont préparé et travaillé à la venue d'une loi proactive. C'est surtout la FTQ comme centrale et le SCFP, le plus gros syndicat canadien, qui ont été le fer de lance de ce mouvement tant sur les plans technique, politique que de la négociation collective. Il faut savoir que des syndicats nationaux comme le SCFP ont vécu l'expérience proactive dans d'autres provinces, surtout en Ontario.

Le chapitre 3 "Vue d'ensemble de la loi sur l'équité salariale " comme son titre l'indique, expose méthodiquement et de façon commentée les principaux éléments de la loi. (À lire avant de s'attaquer à la lecture du texte de la loi elle-même !) Les quatre derniers chapitres (4 à 7) reprennent, étape par étape, la démarche prévue par la loi pour concevoir et réaliser un programme d'équité salariale avec ou sans comité d'équité salariale. Notons que le chapitre 4 insiste dès le départ, comme le fait Gazaille dans l'ouvrage précédent, sur l'importance d'avoir une bonne stratégie de communication et les principes qui devraient la guider. Puis on entre dans la démarche proprement dite, pas à pas, avec commentaires rappelant soit des éléments de littérature empirique ou de jurisprudence, soit répétant les mises en garde dans l'interprétation et l'application des articles de la loi pour que prévale continuellement l'esprit de la loi. Certaines interprétations ou certains commentaires paraîtront au lecteur ne pas aller assez loin sur le plan pratique, telle la délicate 
question du fonctionnement et de la prise de décision en comité, ou feront sursauter telle l'affirmation qu'on peut tracer une courbe salariale des emplois à prédominance masculine à partir d'un minimum de trois observations seulement. Rappelons toutefois qu'il s'agit du premier ouvrage sur le sujet en rapport avec la toute récente Loi sur l'équité salariale au Québec et que l'auteure a dû en "faucher large » en peu de temps pour mettre à la disposition des personnes intéressées un outil d'interprétation le plus complet et le mieux documenté possible.

Le dernier chapitre traitant la phase "post équité salariale ", c'est-à-dire le maintien de celle-ci une fois atteinte, présente également un relevé de la littérature empirique connue à ce jour, surtout en rapport avec la loi ontarienne, des conséquences économiques de l'équité salariale dont la fameuse question des coûts pour l'employeur. (Il s'agit essentiellement d'une reprise d'une partie du rapport du groupe de travail sur l'équité salariale, composé de M.-T. Chicha, E. Déom et H.-L. Gosselin, remis à la ministre J.L. Blackburn lequel conserve d'ailleurs toute son actualité comme on l'a vu durant la Commission parlementaire ayant précédé l'adoption du projet de loi.)

Marie-Thérèse Chicha est une universitaire et une chercheure de haut niveau qui connaît bien son sujet et cet ouvrage est incontournable pour le lecteur intéressé par les questions d'équité entre les sexes sur le marché du travail et plus particulièrement les personnes impliquées à un niveau ou à un autre dans une démarche d'équité salariale.

JACQUES MERCIER Université Laval

\section{ISO 9000: une force de management}

par Branimir TODOROV, Montréal : Gaëtan Morin Éditeur, 1997, 194 p., ISBN 289105-641-8

L'ouvrage ISO 9000 s'avère une très bonne source de renseignements pour introduire le lecteur dans le monde complexe des normes d'assurance de la qualité. Dans un style concis et simple le présent livre «vise à expliquer l'introduction, l'implantation et l'actualisation d'un système de management de la qualité sclon une approche originale et dynamique " (p. XI).

Divisé en 19 chapitres s'étalant sur 194 pages, l'ouvrage traite de façon succincte des principaux points suivants :

- les principes de management de la qualité ;

- les systèmes de management de la qualité ISO 9000 ;

- la certification des systèmes ISO 9000 ;

- la configuration, l'implantation et l'amélioration des systèmes ;

- le système QS-9000;
- le programme d'audits internes;

- 150 questions d'audit d'une entreprise industrielle ;

- 150 questions d'évaluation d'une entreprise de services ;

- la série des normes ISO 14000 et le management environnemental ;

- les prix de la qualité ;

- la famille des normes ISO 9000 de l'an 2000 ;

- quelques cas ;

- une riche bibliographie.

Pour sa part, l'auteur Branimir Todorov s'avère très qualifié dans le domaine, tant au plan universitaire (maîtrise en génie mécanique et titulaire d'un diplôme supérieur en management de la qualité de l'Institut de Gestion Sociale de Paris) qu'au plan pratique, notamment en tant qu'expert international et membre du comité technique des normes ISO de Genève. 